\title{
Efeito de diferentes fontes de carboidrato e da privação de alimento sobre aspectos biológicos de Telenomus remus Nixon (Hymenoptera, Scelionidae)
}

\author{
Ana Paula Meirelles ${ }^{1}$, Tatiana Rodrigues Carneiro $^{1} \&$ Odair Aparecido Fernandes ${ }^{1,2}$
}

\begin{abstract}
${ }^{1}$ Departamento de Fitossanidade, Campus de Jaboticabal, Universidade Estadual Paulista. Rodovia Prof. Paulo Donato Castellane, km. 5, 14884-900, Jaboticabal-SP, Brasil. ap.meirelles@hotmail.com; tatianacarneiro@ hotmail.com; oafernandes@fcav.unesp.br ${ }^{2}$ Pesquisador do CNPq - Autor correspondente.
\end{abstract}

\begin{abstract}
Effect of different carbohidrate sources and food deprivation on biological characteristics of Telenomus remus Nixon (Hymenoptera, Scelionidae). The effect of five carbohidrate sources, water and no food (starvation) was evaluated on biological characteristics of T. remus. The treatments were the following: (a) non-fed females, and females fed with (b) distilled water; (c) honey; (d) glucose syrup (Karo®); (e) glucose solution 1M; (f) fructose solution 1M; (g) sucrose solution $1 \mathrm{M}$; (h) glucose+fructose+sucrose 1M; (i) glucose+fructose+sucrose 3M. Spodoptera frugiperda egg masses with ca. 100 eggs $(<24 \mathrm{~h}$ old) were offered to T. remus females. Parasitism, sex ratio, and longevity were evaluated. In relation to parasitism, females fed on honey and glucose, fructose, and sucrose solutions were similarly more efficient whereas non-fed females and females fed only on water showed low levels of parasitism. Females fed on glucose syrup (Karo ${ }^{\circledR}$ ) and glucose+fructose+sucrose $1 \mathrm{M}$ and $3 \mathrm{M}$ presented significantly lower parasitism than the other treaments. There was no significant difference in the sex ratio of the progeny. The sex ratio was ca. 0.57. On average, females were alive for 5 days when fed on any carbohidrate source, whereas females fed on only water and non fedfemales lived only one day. Therefore, honey, glucose, fructose, and sucrose were adequate food sources for $T$. remus females.
\end{abstract}

KEYWORDS. Behavior; biological control; egg parasitoids; food.

RESUMO. Efeito de diferentes fontes de carboidrato e da privação de alimento sobre aspectos biológicos de Telenomus remus Nixon (Hymenoptera, Scelionidae). O efeito de cinco fontes de carboidratos, da água e da ausência de alimentação foi avaliado sobre aspectos biológicos de T. remus. Os tratamentos constituíram-se de: (a) fêmeas não alimentadas, e alimentadas com (b) água destilada; (c) mel puro; (d) xarope de glicose (Karo®); (e) solução de glicose 1M; (f) solução de frutose $1 \mathrm{M}$; (g) solução de sacarose $1 \mathrm{M}$; (h) solução de glicose+frutose+sacarose $1 \mathrm{M}$; (i) solução de glicose+frutose+sacarose $3 \mathrm{M}$. Os alimentos foram disponibilizados durante todo o experimento. Posturas de Sodoptera frugiperda com aproximadamente 100 ovos $(<24 \mathrm{~h})$ foram ofertadas às fêmeas de T. remus. O parasitismo, a razão sexual e a longevidade foram avaliados. No aspecto parasitismo, as fêmeas alimentadas com mel e soluções de glicose, frutose e sacarose mostraram maior eficiência, não diferindo significativamente entre si, enquanto que as fêmeas não alimentadas e alimentadas somente com água apresentaram níveis mais baixos de parasitismo. Já as fêmeas alimentadas com xarope de glicose $($ Karo $®)$, solução de glicose+frutose+sacarose $1 \mathrm{M}$ e $3 \mathrm{M}$ apresentaram parasitismo mais baixo quando comparados com os outros tratamentos, não diferindo significativamente entre si. Quanto à razão sexual, os tratamentos não apresentaram diferenças significativas, mantendo-se em torno de 0,57. No que se refere à longevidade, os insetos mantiveram-se vivos em média 5 dias quando alimentados com qualquer um dos carboidratos, já aqueles alimentados com água ou não alimentados sobreviveram apenas por 1 dia. Logo, conclui-se que o mel, glicose, frutose e sacarose mostramse mais adequados para a alimentação de fêmeas de T. remus.

PALAVRAS-CHAVE. Alimentação; comportamento; controle biológico; parasitóides de ovos.

Diversos parasitóides e predadores são importantes fatores reguladores das populações de Spodoptera frugiperda (Smith) (Lepidoptera, Noctuidae). Destes destacam-se os parasitóides de ovos, como Telenomus remus Nixon (Hymenoptera, Scelionidae), que eliminam a praga antes que ela cause dano à planta, pois atuam efetivamente sobre os ovos, parasitando inclusive aqueles das camadas mais internas (Cruz 1995; Figueiredo et al. 1999). Este inseto tem preferência por ovos de lepidópteros de Noctuidae, com destaque para insetos do gênero Spodoptera (Cave 2000); possui alta capacidade reprodutiva e facilidade de ser criado em laboratório, demonstrando potencial como agente de controle para o uso em programas de controle biológico aplicado (Cave \& Acosta 1999).

De acordo com Vinson (1998), estudos envolvendo o comportamento dos parasitóides que inclui desde a localização do hospedeiro até o seu reconhecimento, aceitação e finalmente o parasitismo, devem preceder qualquer programa de controle biológico. Além disso, deve-se ressaltar a interferência direta que o alimento possui sobre a longevidade, fecundidade (Berti \& Marcano 1991) e capacidade de busca dos parasitóides (Siekmann et al. 2004; Lee \& Heimpel 2007).

As dietas artificiais utilizadas em laboratório para alimentar inimigos naturais variam desde uma simples solução açucarada até alimentos nutricionalmente completos. A complexidade da dieta depende da biologia do predador ou parasitóide a ser manipulado e do objetivo em mantê-los na área ou na planta, atraí-los para determinado alvo ou mesmo estimulá-los para a produção de ovos (Hagen \& Bishop 1979).

Os parasitóides necessitam de uma fonte de carboidrato, 
pois a ausência destas substâncias interfere na fecundidade, na longevidade e no tempo em que as fêmeas gastam para encontrar seu hospedeiro, influenciando seu potencial de colonização (Pratissoli et al. 2004; Tenhumberg et al. 2006). Há casos em que o amadurecimento de ovos da fêmea é dependente deste recurso (Quednau 1967; Olson \& Andow 1998; Riddick 2007). Garcia (1991) relatou que néctar e pólen representam, em muitos casos, as principais fontes de nutrientes exigidos pela fêmea; assim como excretas de alguns hemípteros (honeydew) que também pode representar uma forma de alimento para adultos de parasitóides (Lee et al. 2004). Mediante o fato de que estes recursos nem sempre estão disponíveis e são necessários para a alimentação dos insetos, em programas de controle biológico aplicado, poderia-se alimentar os parasitóides antes de sua liberação ou disponibilizar estas fontes de alimento no campo (Lee \& Heimpel 2008).

As fêmeas de T. remus, alimentadas com mel durante toda a vida apresentaram maior taxa de parasitismo quando comparadas com aquelas que receberam mel apenas por $24 \mathrm{he}$ que viveram em média apenas 4 dias e por aquelas não alimentadas, que se mantiveram vivas por apenas 1 dia (Loffredo, informação pessoal).

Portanto, como a maioria dos parasitóides adultos depende dos carboidratos como fonte de energia (Jervis et al. 1993) e estes alimentos podem aumentar a eficiência desses inimigos naturais (Lee \& Heimpel 2008), objetivou-se com este trabalho verificar os aspectos biológicos de fêmeas do parasitóide $T$. remus quando alimentadas com diferentes fontes de carboidratos e quando privadas de alimento, complementando assim os conhecimentos a respeito deste inseto que pode vir a ser utilizado com sucesso em programas de controle biológico no Brasil.

\section{MATERIALE MÉTODOS}

O experimento foi conduzido no Laboratório de Ecologia Aplicada, da UNESP/FCAV, Jaboticabal, SP, onde fêmeas de $T$. remus foram mantidas e criadas sobre ovos de $S$. frugiperda.

O desempenho reprodutivo e a longevidade de fêmeas de T. remus foram estudados submetendo-se os insetos à alimentação com cinco fontes de carboidrato, com água destilada e à privação de alimento. Foram utilizadas fêmeas recém emergidas ( $<12 \mathrm{~h}$ de idade) e acasaladas. Os tratamentos foram constituídos por (a) fêmeas não alimentadas; alimentadas com (b) água destilada; (c) mel puro; (d) xarope de glicose (Karo®); e com soluções descritas por Wäckers (2001): (e) solução de glicose $1 \mathrm{M}$; (f) solução de frutose $1 \mathrm{M}$; (g) solução de sacarose $1 \mathrm{M}$; (h) solução de glicose+frutose+sacarose $1 \mathrm{M}$; (i) solução de glicose+frutose+sacarose $3 \mathrm{M}$, disponibilizadas desde a emergência até a morte dos insetos. Água, mel e xarope de glicose foram disponibilizados em forma de pequenas gotas colocadas na parede do tubo com auxílio de estilete, enquanto que as soluções foram disponibilizadas com auxílio de pequenos pedaços de algodão umedecidos. $\mathrm{O}$ mel ofertado aos parasitóides foi submetido a análise físico-química, na qual foram avaliadas as quantidades de açúcares e água, bem como a acidez.
Como hospedeiros foram utilizadas posturas de $S$. frugiperda com aproximadamente 100 ovos e até $24 \mathrm{~h}$ de desenvolvimento embrionário, que foram ofertadas às fêmeas de $T$. remus individualizadas em tubos de ensaio de fundo chato $(2 \times 8 \mathrm{~cm})$ contendo os respectivos alimentos, fechados com filme de PVC (policloreto de vinila). A oviposição foi permitida por $24 \mathrm{~h}$ e após este período, as posturas foram transferidas para tubos vazios para posterior avaliação. Durante todo o experimento os tubos contendo as fêmeas e cartelas com posturas de $S$. frugiperda foram mantidos em câmara climatizada a $25 \pm 1{ }^{\circ} \mathrm{C}, 70 \pm 10 \%$ UR e fotofase de $12 \mathrm{~h}$. Diariamente foram feitas observações e retiradas de lagartas eventualmente eclodidas, que poderiam danificar os ovos parasitados.

Utilizaram-se como parâmetros de avaliação a longevidade, a razão sexual da prole e as taxas de parasitismo diária e acumulada que foram expressas em percentagem, sendo estas mensuradas pela contagem dos adultos emergidos sob microscópio estereoscópico. Adotou-se o delineamento experimental inteiramente casualizado, com nove tratamentos e 20 repetições por tratamento (fêmeas nos tubos com seus respectivos alimentos). Os dados foram submetidos a análise de variância e as médias comparadas pelo Teste de Tukey ( $\mathrm{P} \leq$ 0,05 ) utilizando-se o programa SAS (SAS Institute 2004).

\section{RESULTADOS EDISCUSSÃO}

T. remus teve sua longevidade e fecundidade reduzidas na ausência de carboidratos (Tabela I). Isso corrobora a afirmação de que estes compostos constituem parte muito importante na alimentação de diversos adultos de parasitóides e afetam seus aspectos biológicos (Jervis et al. 1993).

Quando fêmeas de T. remus receberam somente água destilada ou não foram alimentadas, a taxa de parasitismo apresentou-se relativamente baixa e não houve diferença significativa entre esses tratamentos (Tabela I). O mesmo ocorre com espécies de Trichogrammatidae, uma vez que a alimentação interfere diretamente no parasitismo e longevidade das fêmeas (Pratissoli et al. 2004; Leatemia et al. 1995).

Para Chrysocharis laricinellae Ratzeburg (Hymenoptera, Eulophidae) (Quednau 1967) e Cotesia marginiventris (Cresson) (Hymenoptera, Braconidae) (Riddick 2007) foi demonstrado que muitas vezes a maturidade sexual está associada à necessidade de ingestão de alimentos ricos em açúcares e aminoácidos, pois fêmeas destes parasitóides alimentadas apresentaram mais óvulos maduros em menos tempo. Contudo, T. remus não mostrou-se dependente de alimentos para a maturidade sexual, pois mesmo fêmeas não alimentadas, apesar de sobreviverem por apenas um dia, ovipositaram e produziram descendentes (Tabela I e Figura 1).

Com relação à longevidade, as fêmeas de $T$. remus alimentadas apenas com água ou privadas de alimentos sobreviveram por um dia, diferindo significativamente dos demais tratamentos, o que comprova a importância do carboidrato como fonte de energia (Tabela I).

Os principais açúcares que compõem o néctar (glicose, frutose e sacarose) parecem ser os carboidratos mais 


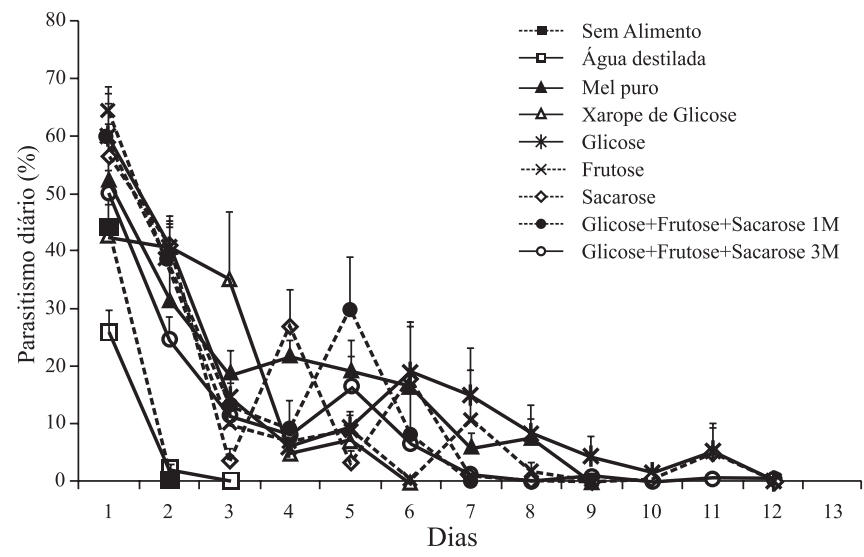

Fig. 1. Parasitismo diário (\%) (+ EPM) de fêmeas de Telenomus remus (Hymenoptera: Scelionidae) sobre ovos de Spodoptera frugiperda (Lepidoptera: Noctuidae) submetidas a diferentes fontes de alimento.

apropriados para a criação de parasitóides, pois não só aumentam a longevidade, como também a capacidade de parasitar mais hospedeiros ao longo da vida (Idris \& Grafius 1995; Wäckers 2001; Hogervorst et al. 2007; Williams III \& Roane 2007). Tais açúcares mostraram-se importantes também para $T$. remus, pois as fêmeas alimentadas com qualquer uma das soluções e com mel, que em sua composição contém $85,48 \%$ de açúcares redutores (glicose + frutose) e 3,57\% de sacarose sobreviveram mais e geraram mais descendentes do que as fêmeas não alimentadas (Tabela I). Logo, estudos envolvendo a alimentação de fêmeas de $T$. remus em condições de campo são importantes, pois caso estes insetos venham a obter uma fonte de alimentação em nectários florais poderiam vir a sobreviver por mais tempo e parasitar mais ovos.

Neste estudo, o mel mostrou-se o alimento mais adequado, pois além de propiciar altos níveis de parasitismo e longevidade, trata-se de um alimento fácil de ser adquirido, pouco dispendioso e que não interfere na biologia dos insetos, o que o classifica como uma dieta adequada para criações massais. Apesar de possuir oligossacarídeos este alimento não mostrou-se diferente dos demais tratamentos com carboidratos (Tabela I). Wäckers (2000, 2001) e Lee et al. (2004) verificaram que o mel, além de conter glicose, frutose e sacarose, também contém outros oligossacarídeos como maltose e rafinose. Os autores salientam que estes oligossacarídeos não são tão apropriados para alimentação dos parasitóides, pois não são absorvidos rapidamente pelo inseto, podem cristalizar em um tempo menor do que a sacarose e ainda, aumentam a vida útil dos insetos em menor escala do que os açúcares como glicose, frutose e sacarose.

Tanto fêmeas alimentadas como não alimentadas apresentaram, no geral, maior número de descendentes fêmeas do que de machos (razão sexual em torno de 0,57), dados semelhantes ao encontrado por Bueno et al. (2008); portanto a alimentação não interferiu na razão sexual de $T$. remus. Leatemia et al. (1995) reportaram que para Trichogramma minutum Riley (Hymenoptera, Trichogrammatidae) a qualidade e, principalmente, a presença do alimento podem afetar a razão sexual, fato também comprovado por Nicoli et al. (2004) ao estudar o comportamento de Trichogramma atopovirilia Oatman \& Platner (Hymenoptera, Trichogrammatidae) em ovos de A. kuehniella.

Quanto ao parasitismo das fêmeas alimentadas, foi observado que a maioria ovipositou nos três primeiros dias de vida (Figura 1), sendo colocado um maior número de fêmeas nos primeiros dias de parasitismo. Tal inversão na razão sexual de parasitóides com a idade da fêmea pode dar-se pela diminuição ou a falta de esperma na espermateca, devido à fêmea copular uma única vez, o que conseqüentemente diminui o número de ovos fertilizados e, assim, o número de fêmeas na próxima geração (Houseweart et al. 1983).

Independente dos açúcares estarem isolados ou em mistura e da concentração destas misturas, T. remus foi capaz de alimentar-se e não houve diferença significativa na longevidade ou no parasitismo. Entretanto, o xarope de glicose (Karo®) não é recomendado para a alimentação do parasitóide, pois não diferenciou-se dos tratamentos nos quais as fêmeas não foram alimentadas, mostrando-se assim um alimento não adequado.

Quando açúcares são misturados ou ofertados em abundância na dieta dos parasitóides, podem ocorrer baixas taxas de parasitismo e razão sexual, que podem ser explicadas por três possíveis motivos: (1) a fonte de açúcar pode ter cristalizado, ou seja, apresentado alta viscosidade, o que dificulta a acessibilidade ao alimento pelo inseto (Wäckers 2000); (2) pela preferência em armazenar nutrientes com alta concentração de açúcares (Boggs 1997), gastando mais tempo

Tabela I. Parasitismo acumulado, razão sexual e longevidade ( \pm EPM) das fêmeas de Telenomus remus mediante diferentes fontes de alimento. Jaboticabal, SP, 2008.

\begin{tabular}{|c|c|c|c|c|c|}
\hline \multirow{2}{*}{$\begin{array}{l}\text { Tratamentos } \\
\text { Sem alimento }\end{array}$} & \multicolumn{2}{|c|}{ Parasitismo Acumulado } & Razão Sexual & \multicolumn{2}{|c|}{ Longevidade (dias) } \\
\hline & $59,9 \pm 6,66$ & $\mathrm{bc}$ & $0,62 \pm 0,08 \quad a$ & $1,0 \pm 0,00$ & $\mathrm{c}$ \\
\hline Água & $34,4 \pm 6,30$ & $\mathrm{c}$ & $0,33 \pm 0,04 \quad a$ & $2,0 \pm 0,00$ & $\mathrm{bc}$ \\
\hline Mel puro & $134,5 \pm 21,46$ & a & $0,57 \pm 0,06 \quad a$ & $4,5 \pm 1,00$ & $\mathrm{a}$ \\
\hline Xarope de Glicose & $109,4 \pm 19,93$ & $a b c$ & $0,53 \pm 0,06 \quad a$ & $3,80 \pm 0,55$ & $\mathrm{a}$ \\
\hline Glicose $1 \mathrm{M}$ & $130,9 \pm 24,51$ & $\mathrm{a}$ & $0,49 \pm 0,07 \quad a$ & $5,60 \pm 1,21$ & $\mathrm{a}$ \\
\hline Frutose $1 \mathrm{M}$ & $134,3 \pm 11,76$ & $\mathrm{a}$ & $0,47 \pm 0,06 \quad a$ & $4,80 \pm 0,68$ & $\mathrm{a}$ \\
\hline Sacarose $1 \mathrm{M}$ & $124,2 \pm 17,00$ & $\mathrm{a}$ & $0,45 \pm 0,07 \quad a$ & $5,00 \pm 1,17$ & $\mathrm{a}$ \\
\hline Glicose+Frutose+Sacarose $1 \mathrm{M}$ & $119,4 \pm 15,70$ & $a b$ & $0,53 \pm 0,08 \quad a$ & $4,70 \pm 1,03$ & $\mathrm{a}$ \\
\hline Glicose + Frutose + Sacarose $3 \mathrm{M}$ & $117,5 \pm 16,68$ & $\mathrm{ab}$ & $0,48 \pm 0,10 \quad a$ & $5,20 \pm 1,18$ & $\mathrm{a}$ \\
\hline
\end{tabular}

Médias seguidas pela mesma letra não diferem entre si pelo Teste de Tukey $(\mathrm{P} \leq 0,05)$. 
e, conseqüentemente, (3) passam mais tempo ingerindo alimento do que parasitando (Azzouz et al. 2004). O parasitismo acumulado pelos insetos alimentados com xarope de glicose (Karo®) não se diferiu dos demais tratamentos, inclusive da ausência de alimento. Logo, a menor viscosidade do xarope de glicose pode ser um dos motivos que levaram as fêmeas alimentadas com este carboidrato à apresentarem menor desempenho.

Conclui-se, portanto, que fêmeas alimentadas com mel, glicose, frutose e sacarose apresentaram maior eficiência considerando-se o parasitismo acumulado, período de oviposição, longevidade e razão sexual, o que pode demonstrar que estes açúcares são fontes de alimentos adequados para criação de T. remus. Cabe investigar se as fêmeas no campo se alimentam e onde podem obter este alimento, uma vez que a cultura do milho não o propicia. Talvez, para aumentar o sucesso em programas de controle biológico, seja importante alimentar as fêmeas antes de liberá-las ou disponibilizar no campo uma fonte de alimento.

Agradecimentos. À Fundação de Amparo à Pesquisa do Estado de São Paulo (FAPESP) pela concessão de bolsa de estudos à primeira autora (Processo ${ }^{\circ}$ 2007/03668-1), às informações fornecidas por Loffredo, A. P. S. (Universidade Federal de São Carlos -UFSCar) e aos dois revisores anônimos pelas sugestões apresentadas.

\section{REFERÊNCIAS}

Azzouz, H.; P. Giordanengo; F. L. Wäckers \& L. Kaiser. 2004. Effects of feeding frequency and sugar concentration on behavior and longevity of the adult aphid parasitoid: Aphidius ervi (Haliday) (Hymenoptera: Braconidae). Biological Control 31: 445-452.

Berti, J. \& R. Marcano. 1991. Effect of time of host absence on parasitism by Trichogramma pretiosum Riley (Hym.: Trichogrammatidae). Boletin de Entomologia Venezolana 6: $5-10$.

Boggs, C. L. 1997. Dynamics of reproductive allocation from juvenile and adult feeding: radiotracer studies. Ecology 78: 192-202.

Bueno, R. C. O. F.; T. R. Carneiro; D. Pratissoli; A. F. Bueno, A. F. \& O. A. Fernandes. 2008. Biology and thermal requirements of Telenomus remus reared on fall armyworm Spodoptera frugiperda eggs. Ciência Rural 38: 1-6.

Cave, R. D. 2000. Biology, ecology and use in pest management of Telenomus remus. Biocontrol News and Information 21: 2126.

Cave, R. D. \& N. M. Acosta. 1999. Telenomus remus Nixon: un parasitoide en el control biológico del gusano cogollero, Spodoptera frugiperda (Smith). Ceiba 40: 215-227.

Cruz, I. 1995. A lagarta-do-cartucho na cultura do milho. Sete Lagoas: EMBRAPA - CNPMS, (Circular Técnica Número 21), 45 p.

Figueiredo, M. L. C.; I. Cruz \& T. M. C. Della Lucia. 1999. Controle integrado de Spodoptera frugiperda (Smith \& Abbott) utilizandose o parasitóide Telenomus remus Nixon. Pesquisa Agropecuária Brasileira 34: 1975-1982.

Garcia, M. A. 1991. Ecologia nutricional de parasitóides e predadores terrestres, p 289-305. In: A. R. Panizzi \& J. R. P. Parra. (eds.). Ecologia nutricional de insetos e suas implicações no manejo de pragas. Brasília. Manole, $359 \mathrm{p}$.

Hagen, K. S. \& G. W. Bishop. 1979. Use of supplemental foods and behavioral chemicals to increase the effectiveness of natural enemies, p. 49-60. In: D. W. Davis; S. Hoyts; J. A. Mc Murtry \& M. T. Aliniazee (eds.). Biological control and insect pest management. Berkeley, University of California Press, 102 p.

Hogervorst, P. A. M.; F. L. Wäckers; J. Romeis. 2007. Effects of honeydew sugar composition on the longevity of Aphidius ervi. Entomologia Experimentalis et Applicata 122: 223-232.

Houseweart, M. W.; D. T. Jennings; C. Welty \& S. G. Southard. 1983 Progeny production by Trichogramma minutum (Hymenoptera: Trichogrammatidae) utilizing eggs for Choristoneura fumiferana (Lepidoptera: Tortricidae) and Sitotroga cerealella (Lepidoptera: Gelechiidae). Canadian Entomologist 115: 1245-1252.

Idris, A. B. \& E. Grafius. 1995. Wildflowers as nectar sources for Diadegma insulare (Hymenoptera: Ichneumonidae), a parasitoid of diamondback moth (Lepidoptera: Yponomeutidae). Environmental Entomology 24: 1726-1735.

Jervis, M. A.; N. A. C. Kidd; M. G. Fitton; T. Huddleston \& H. A Dawah. 1993. Flower-visiting by hymenopteran parasitoids. Journal of Natural History 27: 67-105.

Leatemia, J. A.; J. E. Laing \& J. E. Corrigan. 1995. Effects of adult nutrition on longevity, fecundity, and offspring sex ratio of Trichogramma minutum Riley (Hymenoptera: Trichogrammatidae). The Canadian Entomologist 127: 245254.

Lee, J. C.; G. E. Heimpel \& G. L. Leibee. 2004. Comparing floral nectar and aphid honeydew diets on the longevity and nutrients levels of a parasitoid wasp. Entomologia Experimentalis at Applicata 111: $189-199$

Lee, J. C. \& G. E. Heimpel. 2007. Sugar feeding reduces short-term activity of a parasitoid wasp. Physiological Entomology 32: 99-103.

Lee, J. C. \& G. E. Heimpel. 2008. Floral resources impact longevity and oviposition rate of a parasitoid in the field. Journal of Animal Ecology 77: 565-572.

Nicoli, E. M.; D. Pratissoli; E. F. Reis \& H. F. Santos. 2004. Viabilidade e razão sexual de Trichogramma atopovirilia Oatman \& Platner, 1983 (Hymenoptera: Trichogrammatidae) sob influência do hospedeiro Anagasta kuehniella (Zeller, 1879) (Lepidoptera: Pyralidae) em condições de laboratório. Entomologia y Vectores 11: $521-533$.

Olson, D. M. \& D. A. Andow. 1998. Larval Nutrition effects on longevity and fecundity of female Trichogramma nubilale Ertle \& Davis (Hymenoptera: Trichogrammatidae). Environmental Entomology 27: 508-514.

Pratissoli, D.; H. N. de Oliveira; S. M. J. Vieira; R. C. Oliveira \& H. B. Zago. 2004. Efeito da disponibilidade de alimento nas características biológicas de Trichogramma galloi Zucchi (Hymenoptera: Trichogrammatidae). Revista Brasileira de Entomologia 48: 101-104.

Quednau, F. W. 1967. Notes on mating behaviour and oviposition of Chrysocharis laricinellae, a parasitoid of the larch caseborer. Canadian Entomologist 99: 326-331.

Riddick, E. W. 2007. Influence of honey and maternal age on egg load of lab-cultured Cotesia marginiventris. BioControl 52: 613-618.

SAS Institute. 2004. SAS/STAT user's guide, release 9.1 ed. SAS Institute, Cary, NC.

Siekmann, G.; M. A. Keller \& B. Tenhumberg. 2004. The sweet tooth of adult parasitoid Cotesia rubecula : ignoring hosts for nectar? Journal of Insect Behavior 17: 459-475.

Tenhumberg, B.; G. Siekmann \& M. A. Keller. 2006. Optimal time allocation in parasitic wasps searching for hosts and food. Oikos 113: $121-131$.

Vinson, S. B. 1998. The general host selection behavior of parasitoid Hymenoptera and a comparison of initial strategies utilized by larvaphagous and oophagous species. Biological Control 11: 7996.

Wäckers, F. L. 2000. Do oligosaccharides veduce the suitability of honeydew for predators and parasitoids? A further facet to the function of insect-synthesized honeydew sugars. Oikos 90: 197201.

Wäckers, F. L. 2001. A comparison of nectar- and honeydew sugars with respect to their utilization by the hymenopteran parasitoid Cotesia glomerata. Journal of Insect Physiology 47: 10771084 .

Williams III, L. \& T. M. Roane. 2007. Nutritional ecology of a parasitic wasp: Food source affects gustatory response, metabolic utilization, and survivorship Journal of Insect Physiology 53: 1262-1275.

Recebido em 12/05/2008; aceito em 27/12/2008 